\title{
New Test of Supernova Electron Neutrino Emission using Sudbury Neutrino Observatory Sensitivity to the Diffuse Supernova Neutrino Background
}

\author{
John F. Beacom ${ }^{1,2, *}$ and Louis E. Strigari ${ }^{1, \text { 田 }}$ \\ ${ }^{1}$ Department of Physics, The Ohio State University, Columbus, OH 43210, USA \\ ${ }^{2}$ Department of Astronomy, The Ohio State University, Columbus, OH 43210, USA
}

(Dated: 18 August 2005)

\begin{abstract}
Supernovae are rare nearby, but they are not rare in the Universe, and all past core-collapse supernovae contributed to the Diffuse Supernova Neutrino Background (DSNB), for which the near-term detection prospects are very good. The Super-Kamiokande limit on the DSNB electron antineutrino flux, $\phi\left(E_{\nu}>19.3 \mathrm{MeV}\right)<1.2 \mathrm{~cm}^{-2} \mathrm{~s}^{-1}$, is just above the range of recent theoretical predictions based on the measured star formation rate history. We show that the Sudbury Neutrino Observatory should be able to test the corresponding DSNB electron neutrino flux with a sensitivity as low as $\phi\left(22.5<E_{\nu}<32.5 \mathrm{MeV}\right) \simeq 6 \mathrm{~cm}^{-2} \mathrm{~s}^{-1}$, improving the existing Mont Blanc limit by about three orders of magnitude. While conventional supernova models predict comparable electron neutrino and antineutrino fluxes, it is often considered that the first (and forward-directed) SN 1987A event in the Kamiokande-II detector should be attributed to electron-neutrino scattering with an electron, which would require a substantially enhanced electron neutrino flux. We show that with the required enhancements in either the burst or thermal phase $\nu_{e}$ fluxes, the DSNB electron neutrino flux would generally be detectable in the Sudbury Neutrino Observatory. A direct experimental test could then resolve one of the enduring mysteries of SN 1987A: whether the first Kamiokande-II event reveals a serious misunderstanding of supernova physics, or was simply an unlikely statistical fluctuation. Thus the electron neutrino sensitivity of the Sudbury Neutrino Observatory is an important complement to the electron antineutrino sensitivity of Super-Kamiokande in the quest to understand the DSNB.
\end{abstract}

PACS numbers: 97.60.Bw, 98.70.Vc, 95.85.Ry, 14.60.Pq

\section{INTRODUCTION}

The core-collapse death of a massive star and the subsequent type II optical supernova occurs at a rate of $\sim 1$ per second in the Universe, each releasing a prodigious blast of $\sim 10^{58}$ neutrinos and antineutrinos. While a burst of many neutrino events would be detected from a Milky Way supernova [1], the expected occurrence rate is only $\sim 3$ per century [2]. For supernova as far away as $10 \mathrm{Mpc}$, it should be possible to reliably detect neutrinos just one or two at a time, perhaps as often as once per year, with present and proposed detectors [3]. However, most supernovae are vastly farther, such that the expected number of neutrino events detected per supernova is $\ll 1$. When weighted with the total supernova rate, however, the prospects are encouraging for the detection of the Diffuse Supernova Neutrino Background (DSNB). The Super-Kamiokande (SK) limit [4] on the flux of DSNB electron antineutrinos, $\phi\left(E_{\nu}>19.3 \mathrm{MeV}\right)<1.2$ $\mathrm{cm}^{-2} \mathrm{~s}^{-1}$, is just above the range of recent theoretical predictions [5, 6, 7, 8]. In particular, the calculation based on the most recent astronomical data predicts that the DSNB is on the verge of detectability [8].

Searching for the DSNB signal requires the detection of an excess event rate over backgrounds, exploiting the different energy spectra of the signal and backgrounds.

\footnotetext{
*Electronic address: beacom@mps.ohio-state.edu
}

${ }^{\dagger}$ Electronic address: strigari@mps.ohio-state.edu
Due to the near-isotropy of the scattered positrons in the reaction $\bar{\nu}_{e}+p \rightarrow e^{+}+n$ and the large distances to the supernovae, the detections of DSNB events will not be associated with particular optical bursts. If SuperKamiokande is enhanced by the addition of gadolinium, as proposed by Beacom and Vagins, the detector backgrounds would be greatly reduced, allowing the clean detection of as many as 6 events per year 9, 10]. Detection of the DSNB may be the first detection of neutrinos from beyond $1 \mathrm{Mpc}$, and the second detection of supernova neutrinos. In just a few years, the yield from SN 1987A could be surpassed. This will provide valuable insight into supernova physics, neutrino properties, and the history of cosmological massive star formation.

In the following, we discuss the prospects for detection of flavors of the DSNB other than $\bar{\nu}_{e}$, which is the dominant yield in SK. Specifically, we propose using the Sudbury Neutrino Observatory (SNO) to study the DSNB electron neutrino flux. SNO has a unique sensitivity to the electron neutrino flux though the charged-current interaction with deuterons, $\nu_{e}+d \rightarrow e^{-}+p+p$. By combining this charged-current reaction with neutrino-electron elastic scattering and the neutral-current breakup reaction $\nu+d \rightarrow \nu+n+p$, SNO has studied in detail the ${ }^{8} \mathrm{~B}$ solar neutrino spectrum [11], with analysis of the hep spectrum forthcoming. Using the theoretical hep spectrum and the measured atmospheric backgrounds above $20 \mathrm{MeV}$ at SK [4], we estimate the DSNB backgrounds at SNO. We then show that SNO could very likely reach a sensitivity as low as $\phi\left(22.5<E_{\nu}<32.5 \mathrm{MeV}\right) \simeq$ $6 \mathrm{~cm}^{-2} \mathrm{~s}^{-1}$. This would improve the existing limit from 
Mont Blanc by about three orders of magnitude [12], and provide an important complement to the SK $\bar{\nu}_{e}$ limit. In addition to the importance of studying the $\nu_{e}$ emission for supernova models, it is also crucial for testing neutrino mixing, as stressed by Lunardini and Smirnov [13].

There is an additional motivation to test the DSNB $\nu_{e}$ flux separately from $\bar{\nu}_{e}$, stemming from the observed angular distribution of the SN 1987A data. Two wellstudied features of this data that still stand out are that the first event in the Kamiokande-II detector was scattered forward, and the time-integrated angular distributions in both the Kamiokande-II and IMB detectors were both generally more forward and less isotropic than expected from $\bar{\nu}_{e}+p \rightarrow e^{+}+n$ events alone. Both features have typically been explained as either an increase in the neutrino-electron scattering rate beyond theoretical predictions, or a statistical fluctuation [14]. With the SN 1987A data alone, this debate cannot be resolved.

Increasing the $\nu_{e}$ flux to the extent that these features of the SN 1987A data were a probable outcome is in conflict with standard models of supernova neutrino emission. However, given the fact that the models generally fail to produce successful explosions, the possibility of a serious misunderstanding of supernovae, perhaps due to new physics, cannot be excluded. Here we point out that such an increase is testable, in that it would generally lead to the DSNB $\nu_{e}$ flux being large enough for SNO to detect. The absence of a signal would greatly strengthen the case that these features of the angular distribution of the SN 1987A data were due to a statistical fluctuation. Resolution of this point is also important for the future interpretation of the SK DSNB $\bar{\nu}_{e}$ results.

In Section III we review the DSNB predictions and limits; in Section [III we calculate the capabilities of SNO to detect the DSNB $\nu_{e}$ flux; in Section IV we apply these results to discuss the future constraints on the electron neutrino emission from SN1987A, and then we finish with our conclusions.

\section{PRESENT PERSPECTIVE ON THE DSNB}

\section{A. Emission per Supernova}

Stars greater than $\sim 8 M_{\odot}$ are able to burn elements by successive nuclear fusion reactions until iron is reached, beyond which point no further energy generation is possible [15. Once the core has formed about a Chandrasekhar mass of iron, not even electron degeneracy pressure can support it under the weight of the stellar envelope, and it collapses until it reaches the density of nuclear matter, bounces, and forms an outgoing shock. If energetic enough, the shock will eject the envelope and cause the optical supernova, leaving behind a neutron star; if not, the core and envelope will collapse into a black hole. In either case, the $3 \times 10^{53} \mathrm{erg}$ of gravitational binding energy release is dominantly radiated away by all flavors of neutrinos and antineutrinos over about $10 \mathrm{~s}$ (if black hole formation occurs sooner than this, then the neutrino emission is less). It is usually assumed that this total energy release $E_{\nu}^{\text {tot }}$ is shared more or less equally among the six flavors of neutrinos and antineutrinos. For recent updates and reviews on supernova neutrino emission, see Refs. 15, 16]. The spectroscopically classified type II, Ib, and Ic supernovae (hereafter SNII) result from massive star core collapse, and lead to the emission of $\sim 10^{58}$ neutrinos and antineutrinos. Type Ia supernovae (hereafter SNIa) result from the sudden ignition of a white dwarf accreting material from a companion, and are not accompanied by comparable neutrino emission.

In core-collapse supernovae, neutrinos must diffuse out of the proto-neutron star, so that they should decouple with nearly thermal spectra, characteristic of their surface of last scattering. Since $\nu_{\mu}$ and $\nu_{\tau}$ and their antiparticles only have neutral-current interactions at these low energies, they should decouple at the smallest radius and largest temperature. Noting the charged-current interactions of $\nu_{e}$ and $\bar{\nu}_{e}$, and that the medium is neutron-rich, one conventionally expected a hierarchy of temperatures like $T_{\nu_{e}} \simeq 4 \mathrm{MeV}, T_{\bar{\nu}_{e}} \simeq 5 \mathrm{MeV}$, and $\mathrm{T}_{\nu_{\mu}} \simeq 8 \mathrm{MeV}$. More recent work indicates both lower expected temperatures, as well as lower differences between flavors [16]. Assuming equipartition of the total energy among the six flavors, the spectrum per flavor is approximately

$$
\frac{d N}{d E}(E)=\frac{E_{\nu}^{\text {tot }}}{6} \frac{120}{7 \pi^{4}} \frac{E^{2}}{T^{4}}\left[\exp \left(\frac{E}{T}\right)+1\right]^{-1} .
$$

Neutrino oscillations may mix the spectra of different flavors, and importantly, can increase the average energy of the more easily detectable $\bar{\nu}_{e}$ and $\nu_{e}$ (for example, see Ref. [17]), and we return to this point below.

\section{B. Integration over Past Supernovae}

It has been known for some time that the star formation rate was larger in the past, and in particular, was about an order of magnitude larger at redshift $z \simeq 1$ than it is today, likely increasing more slowly at larger redshifts, and first turning on at an uncertain redshift $z \gtrsim 5$ (these data have been comprehensively reviewed by Hopkins [18]). In the past year, these results have been markedly improved by new data, especially from the GALEX ultraviolet satellite 19]. These and other recent data 20, 21, 22], while confirming the basic picture above, also indicate that the absolute rates are on the high side of past estimates, due to larger (and better understood) corrections for obscuration by dust.

Interestingly, this recent trend in astronomical analyses to favor larger dust corrections and larger star formation rates can be very usefully constrained by the upper limits on the DSNB. In Ref. [8], Strigari et al. developed a "Concordance Model" for the star formation and supernova rates which accounts for these and other data. This model is characterized by the star formation rate 
per comoving cubic Mpc today, $R_{\mathrm{SFR}}(z=0)=0.02$ $\mathrm{Mpc}^{-3} \mathrm{yr}^{-1}$, and the growth rate $R_{\mathrm{SFR}}(z) / R_{\mathrm{SFR}}(z=$ $0)=(1+z)^{\beta}$, with $\beta=2$, assumed to hold to $z=1$, after which a constant rate was assumed. These choices of $R_{\mathrm{SFR}}(z=0)$ and $\beta$ are slightly conservative, relative to the GALEX data (see Fig. 1 of Ref. [8]). For larger $z$, the neutrinos are typically redshifted below the detector threshold, and so have relatively little effect on the result. Taking into account the stellar initial mass function, this converts to a core-collapse rate of $R_{\mathrm{SNII}}(z=0)=2.5 \times 10^{-4} \mathrm{Mpc}^{-3} \mathrm{yr}^{-1}$, with the same functional form, since SNII stellar lifetimes are short [8]. An important test of the Concordance Model is that it agrees with measurements of the SNII rate (and the SNIa rate too), confirming the choices of conversion factors, and requiring that the fraction of failed supernovae directly forming black holes [23] must be relatively small [8]. The neutrino emission parameters assumed were $3 \times 10^{53} \mathrm{erg}$, equipartitioned among flavors, and normal-hierarchy mixing of $T=5 \mathrm{MeV} \bar{\nu}_{e}$ with $T=8$ $\mathrm{MeV} \bar{\nu}_{\mu}$ and $\bar{\nu}_{\tau}$. For smaller temperatures and/or differences between temperatures, the predicted flux would be somewhat less.

Using the Concordance Model [8] results for the SNII rate convolved with the neutrino emission per supernova $d N / d E$, the expected DSNB differential flux $d \phi / d E$ is

$$
\frac{d \phi(E)}{d E}=\int R_{\mathrm{SNII}}(z) \frac{d N(E(1+z))}{d E}(1+z) \frac{d t}{d z} d z,
$$

where the integral runs from redshift zero to at least $z \simeq$ 1 , beyond which there is little contribution. To determine $d t / d z$, we use a $\Lambda$-CDM cosmology, $\Omega_{\mathrm{M}}=1-\Omega_{\Lambda}=0.3$, and $H_{0}=70 \mathrm{~km} \mathrm{~s}^{-1} \mathrm{Mpc}^{-3}$, so that

$$
\left|\frac{d t}{d z}\right|=\frac{1}{\mathrm{H}_{0}(1+z) \sqrt{\Omega_{\mathrm{M}}(1+z)^{3}+\Omega_{\Lambda}}} .
$$

Our results are in good general agreement with other recent calculations up to different choices of the inputs [5, 6, 7]; these results and others are nicely reviewed by Ando and Sato [24]. The key input is the star formation rate at redshift zero, and the most recent astronomical measurements used in Ref. [8] are both larger and more precise than those used in Refs. [5, 6, 7, 24]. In Fig. [1] we show predictions for the DSNB $\nu_{e}$ fluxes, for a few possible choices of the $\nu_{e}$ temperature, without including any specific neutrino oscillation scenario yet.

\section{Existing Limits on the DSNB}

The SK upper limit on the DSNB flux of $\bar{\nu}_{e}$ is by far the most stringent for any flavor, and is $1.2 \mathrm{~cm}^{-2} \mathrm{~s}^{-1}$ for energies above $19.3 \mathrm{MeV}[4]$. (With increasing energy, the signal falls and the background rises, and so the flux at energies beyond about $30 \mathrm{MeV}$ is less important.) The detection channel in SK is inverse beta decay on free proton targets, $\bar{\nu}_{e}+p \rightarrow e^{+}+n$, and in

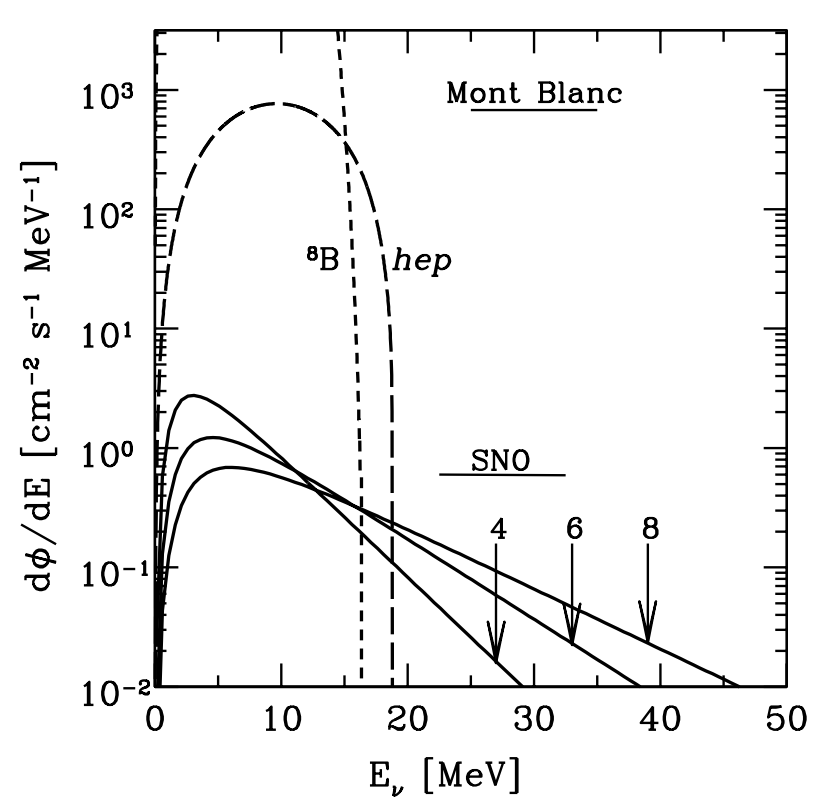

FIG. 1: Predictions for the DSNB $\nu_{e}$ spectra assuming $T=4,6$, or $8 \mathrm{MeV}$ (solid curves), along with the approximate published Mont Blanc limit and the projected SNO sensitivity (solid lines). The solar neutrino $\nu_{e}$ spectra, which are irreducible backgrounds for this study, are also shown. The atmospheric neutrino fluxes are not shown here, but their effects on the detectable spectra are shown in Fig. 2

the existing analysis, only the positron is detected [4]. At the present level of uncertainty and low statistics, the recoil-order corrections to the cross section and kinematics 25] can be ignored, so that $E_{e} \simeq E_{\nu}-1.3 \mathrm{MeV}$, where $E_{e}$ is the positron total energy, following the SK convention. Other singles rates in the same energy range as the signal create serious backgrounds. Below $E_{e}=18$ $\mathrm{MeV}$, uncut muon-induced beta radioactivities and solar neutrino events overwhelm the DSNB signal. At higher energies, the dominant background is from the decays of sub-Čerenkov muons, produced by atmospheric $\nu_{\mu}$ and $\bar{\nu}_{\mu}$ interacting inside the fiducial volume. These nonrelativistic muons quickly lose energy and decay at rest, with the relativistic electrons and positrons following the well-known Michel spectrum. Since their spectrum is known, and the total rate can be normalized from the data, the results are nearly independent of atmospheric neutrino flux uncertainties. The atmospheric $\nu_{e}$ and $\bar{\nu}_{e}$ fluxes create a smaller background that can be well fit at energies above the Michel peak. Figure 2 of Ref. [4] is a clear illustration of these backgrounds and their large impact on the DSNB signal sensitivity. Importantly, recent DSNB calculations [5, 6, 7, 8, 24] are not far below the SK limit, and the Concordance Model result based on the latest astronomical data is especially close [8].

The prospects for the future may be even brighter. Beacom and Vagins proposed that if SK were enhanced by the addition of dissolved gadolinium trichlo- 
ride $\left(\mathrm{GdCl}_{3}\right)$, then it would be possible to tag the neutrons produced in the signal reaction, greatly reducing the background rates [9]. Gadolinium has a huge cross section for radiative neutron capture, and the produced gamma rays would Compton scatter electrons, producing detectable Čerenkov light. With much-reduced backgrounds, a more favorable energy range could be used (beginning near $10 \mathrm{MeV}$ ), and SK could cleanly detect as many as 6 DSNB $\bar{\nu}_{e}$ events per year; see Fig. 1 of Ref. 9$]$. This could lead to the first detection of DSNB neutrinos. While gadolinium compounds have been commonly used in oil-based detectors, Ref. [9] was the first to propose that dissolved gadolinium could be added to a waterbased detector, and that this is the only cost-feasible option to develop a neutron-sensitive detector as large as SK, or ultimately at the 1-Mton scale [9]. The research and development work continues to be very encouraging, supporting the claims that the required standards of solubility, ease of use, water transparency, radiopurity, effects on detector materials, cost, and safety will be met [9, 10]. As a full system test, in late 2005 Vagins will add $\mathrm{GdCl}_{3}$ to the 1-kton water-Cerenkov detector at KEK (a scale model of SK, and a former near detector of the K2K long-baseline neutrino experiment [26]).

The only published limits on the DSNB fluxes of other flavors come from the Mont Blanc experiment [12]. Their limit on $\nu_{e}$ flux is $\phi\left(25<E_{\nu}<50 \mathrm{MeV}\right)<6.8 \times 10^{3}$ $\mathrm{cm}^{-2} \mathrm{~s}^{-1}$, and is based on $\nu_{e}$ charged-current interactions with ${ }^{12} \mathrm{C}$. Since the DSNB spectra are quickly falling, even when weighted with the detection cross section, nearly all of their sensitivity would have come from the beginning of the above energy range. To make a more direct comparison with the sensitivity we derive for SNO, we simply assume that the Mont Blanc limit also applies to an interval of width $10 \mathrm{MeV}$, i.e., from 25 to $35 \mathrm{MeV}$. In Fig. 1 we show both the Mont Blanc limit and the projected SNO sensitivity as if they were constant over $10 \mathrm{MeV}$, which is an approximation (e.g., note Fig. 2).

There is also a Mont Blanc limit on the $\nu_{\mu}$ and $\nu_{\tau}$ flux, based on neutral-current interactions with ${ }^{12} \mathrm{C}$, and this is $\phi\left(20<E_{\nu}<100 \mathrm{MeV}\right)<3 \times 10^{7} \mathrm{~cm}^{-2} \mathrm{~s}^{-1}$, with a similar limit for $\bar{\nu}_{\mu}$ and $\bar{\nu}_{\tau}$. These are very weak limits (for comparison, the solar ${ }^{8} \mathrm{~B}$ flux is $5 \times 10^{6} \mathrm{~cm}^{-2}$ $\mathrm{s}^{-1}$ ), and could be greatly improved. For example, a DSNB flux as large as the Mont Blanc limit would cause a huge excess of neutral-current deuteron breakup events in SNO, at least $10^{3}$ neutrons per day. It may be sufficient to set limits on the fluxes of DSNB $\bar{\nu}_{e}$ and $\nu_{e}$ and use knowledge of the neutrino mixing angles to deduce limits on the DSNB fluxes of $\nu_{\mu}, \nu_{\tau}$, and their antiparticles.

\section{DSNB DETECTION IN SNO}

\section{A. Detection of $\nu_{e}+d \rightarrow e^{-}+p+p$}

SNO, the first water-Čerenkov detector to use deuterons as a target for astrophysical neutrinos, is based on 1 kton of heavy water, $\mathrm{D}_{2} \mathrm{O}[27,28]$. The deuterons are targets for the charged-current reactions $\nu_{e}+d \rightarrow$ $e^{-}+p+p$ and $\bar{\nu}_{e}+d \rightarrow e^{+}+n+n$; electrons and positrons are detected by their Čerenkov light, as in light-water detectors like SK. Additionally, SNO has the ability to detect neutrons, so that it can measure the neutral-current reactions $\nu+d \rightarrow \nu+p+n$ and $\bar{\nu}+d \rightarrow \bar{\nu}+p+n$, sensitive to all flavors of neutrinos and antineutrinos, and separate them from the two charged-current reactions. Three neutron detection techniques have been used in SNO: radiative neutron capture on deuterons in the pure $\mathrm{D}_{2} \mathrm{O}$ phase, radiative neutron capture on chlorine in the $\mathrm{D}_{2} \mathrm{O}$ plus dissolved $\mathrm{NaCl}$ phase, and capture on discrete ${ }^{3}$ He-based neutron counters at present [27, 28]. In all cases, neutrons are simply counted, with no energy or direction information available.

In a search for $\nu_{e}$, SNO has the advantage of the large and spectral (good fidelity between incoming neutrino and electron energy) cross section on deuterons; SK depends on the much less favorable neutrino-electron scattering cross section. In contrast, in a search for $\bar{\nu}_{e}$, SK has the advantage of a much larger detector size, as well as a more favorable cross section on free protons. The other key features of SNO are the existence of a good neutral-current detection channel and the ability to detect neutrons. While the former is unique to $\mathrm{D}_{2} \mathrm{O}$, SK may soon have the latter ability [9, 10], leveraged by its much greater fiducial mass of 22.5 kton.

Other authors have considered the SNO sensitivity to the DSNB $\bar{\nu}_{e}$, noting that the very clean signal coincidence of a positron and two separate neutrons should allow better background rejection than in SK [29, 30]. With limited exposure and low neutron detection efficiency (in the pure $\mathrm{D}_{2} \mathrm{O}$ phase), the SNO results so far are not very restrictive, but significant improvements are expected [31].

What we are proposing here, which has not been noted before, is that SNO should be able to make a unique contribution by exploiting its sensitivity to DSNB $\nu_{e}$. Since this will be just a singles search (only the electron in the final state is detectable), consideration of backgrounds will be crucial.

Recent calculations of the neutrino-deuteron cross sections are given in Refs. 32, 33]; in this energy range, the $\nu_{e}$ cross section is $\simeq 65 \%$ larger than for $\bar{\nu}_{e}$, and a factor $\simeq 2$ less than the inverse beta cross section on free protons. The threshold for the $\nu_{e}+d$ interaction is $1.4 \mathrm{MeV}$, less than the $4.0 \mathrm{MeV}$ for $\bar{\nu}_{e}+d$ or $1.8 \mathrm{MeV}$ for $\bar{\nu}_{e}+p$. In the $\nu_{e}$ channel, while the maximum electron (total $=$ kinetic plus mass) energy is $E_{e}=E_{\nu}-0.9 \mathrm{MeV}$, the peak in the differential cross section is lower [32, 33], and so we use $E_{e}=E_{\nu}-2.5 \mathrm{MeV}$ to assign energies. For the present purposes, a delta function with these kinematics is an adequate approximation to the full differential cross section in the relevant energy range, and makes interpretation of the results more straightforward. The 
total event rate is

$$
R_{\nu}=N_{d} T \int \frac{d \phi\left(E_{\nu}\right)}{d E_{\nu}} \frac{d \sigma\left(E_{\nu}, E_{e}\right)}{d E_{e}} d E_{\nu} d E_{e}
$$

where $N_{d}=6 \times 10^{31}$ is the number of deuterons per kton of $\mathrm{D}_{2} \mathrm{O}$, and $T$ is the exposure time. In making our estimate of the SNO DSNB sensitivity, we assume that the total exposure will be 5 kton-yr at full efficiency. We emphasize that in order to more accurately determine the true sensitivity, a comprehensive study by the SNO collaboration is needed.

In Fig. 2] we show our estimates of the DSNB $\nu_{e}$ detection spectra for three chosen temperatures. SNO is less sensitive than SK, and thus can only detect the DSNB if the flux is larger than expected. We have thus renormalized our standard predictions according to our detection criterion, justified in more detail below, of 1.6 expected signal events in 5 years with detected energy $E_{e}$ between 20 and $30 \mathrm{MeV}$. A detailed discussion of the backgrounds is needed, and we turn to that next.

\section{B. Detector Backgrounds}

In Fig. 2] we show the renormalized DSNB $\nu_{e}$ spectra, along with our estimates of the most important background processes: solar neutrinos and sub-Čerenkov muons produced by atmospheric neutrinos. As we will show, the possible SNO sensitivity to DSNB $\nu_{e}$ is several times worse than the existing SK limit on DSNB $\bar{\nu}_{e}$; therefore, we ignore DSNB $\bar{\nu}_{e}$ interactions as a possible background.

Since the proposed detection channel is $\nu_{e}+d \rightarrow$ $e^{-}+p+p$, below about $20 \mathrm{MeV}$ solar neutrinos provide an overwhelming background, even taking into account the fact that neutrino mixing reduces the flux of $\nu_{e}$. The solar neutrino fluxes [34] are shown in Fig. 1] At the highest energies, the dominant background is from the solar hep reaction $\left({ }^{3} \mathrm{He}+p \rightarrow{ }^{4} \mathrm{He}+e^{+}+\nu_{e}\right)$ [35]; even though its flux is $\sim 10^{3}$ times smaller than the solar ${ }^{8} \mathrm{~B}$ beta decay 36. flux, its endpoint is about $19 \mathrm{MeV}$, about $3 \mathrm{MeV}$ higher than for ${ }^{8} \mathrm{~B}$. Since we only consider energies above $20 \mathrm{MeV}$, this remains true even when energy resolution [11] is taken into account, as we do. There is no possibility of significantly reducing this background, since it is the same detection reaction as the signal, and the angular distribution is only weakly backward (see Fig. 3 of Ref. [25]). Therefore we neglect consideration of all other backgrounds, and the precise details of the signals, below $20 \mathrm{MeV}$. Additionally, we neglect the diffuse $\nu_{e}$ flux made by all of the stars in the Universe before they end their lives (some as supernovae), since this is buried by the solar $\nu_{e}$ flux 37].

In the SK DSNB $\bar{\nu}_{e}$ search, the most significant background at high energies is from the electrons and positrons produced by sub-Čerenkov muons decaying at rest. These muons are invisible since they are produced inside the detector fiducial volume with non-relativistic

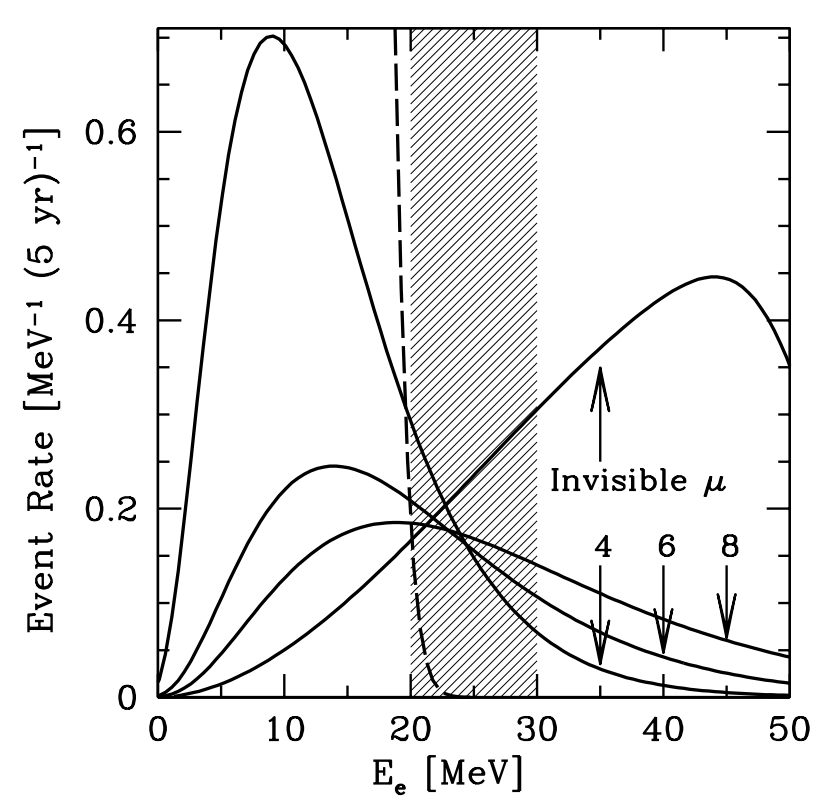

FIG. 2: The DSNB $\nu_{e}$ signal spectra in SNO for a 5 ktonyr exposure, each renormalized to give an expectation of 1.6 signal events in the electron energy range of $20-30 \mathrm{MeV}$ (shaded). The renormalization factors for each temperature are given in Table \ Below $20 \mathrm{MeV}$, solar neutrino interactions (the hep flux is shown with the dashed line) and other backgrounds are overwhelming. Above $30 \mathrm{MeV}$, the subCerenkov (invisible) muon and other atmospheric neutrino backgrounds are too large. The energy resolution of SNO has been taken into account. In the irrelevant region below 20 $\mathrm{MeV}$, the signal curves are subject to some approximations in the calculation, and should not be taken too literally.

initial energies, and they quickly stop; their decay spectrum is simply the well-known Michel spectrum. Since SK has measured the rate of these decays, and their spectrum is fixed, this greatly reduces the dependence on atmospheric neutrino flux predictions. The invisible muons are produced by low-energy atmospheric $\nu_{\mu}$ and $\bar{\nu}_{\mu}$, primarily interacting with bound neutrons and protons in oxygen nuclei. After correcting for detector efficiency, the measured rate in SK is about 60 events per year inside the 22.5 kton fiducial volume 4]. Taking into account vacuum neutrino oscillations of $\nu_{\mu}$ into $\nu_{\tau}$ (and their antiparticles), this is in good agreement with expectations [4]. There are also background events due to very low-energy atmospheric $\nu_{e}$ and $\bar{\nu}_{e}$, also primarily interacting with bound nucleons. Since their rate is much smaller than the rate due to decaying invisible muons, especially below $40 \mathrm{MeV}$ [4], we do not consider them further.

In the energy range $20-30 \mathrm{MeV}$, the efficiencycorrected rate of invisible muon decays in SK is about $0.5 / \mathrm{kton} / \mathrm{yr}$. To estimate the rate of invisible muon decays in SNO, we have to correct for the fact that SK and SNO are at different latitudes. In the relevant neutrino energy range, near $200 \mathrm{MeV}$, Ref. 38] suggests a 
TABLE I: For each assumed temperature, we list the total DSNB flux and the flux just in the energy range $22.5-32.5$ $\mathrm{MeV}$, both in units of $\mathrm{cm}^{-2} \mathrm{~s}^{-1}$. The last column gives the factor by which the flux would need to be increased to meet our detection criteria.

\begin{tabular}{cccc}
\hline \hline $\mathrm{T}$ & $\phi\left(\right.$ all $\left.E_{\nu}\right)$ & $\phi(22.5-32.5 \mathrm{MeV})$ & $\phi_{\text {sens. }} / \phi_{\text {pred. }}$ \\
\hline 4 & 20 & 0.2 & 32 \\
5 & 16 & 0.4 & 15 \\
6 & 14 & 0.6 & 9 \\
7 & 12 & 0.8 & 7 \\
8 & 10 & 0.9 & 6 \\
\hline \hline
\end{tabular}

correction of 1.8, while Ref. [39] suggests 1.5. To be conservative, we estimate that the rate will be a factor of 2 larger at the location of SNO than in SK. We assume that events with a final-state neutron can be tagged, and that about half of the events produced on oxygen can be tagged by a nuclear de-excitation gamma from the residual ${ }^{15} \mathrm{O}$ or ${ }^{15} \mathrm{~N}$ [40]; together these lead to a reduction factor in the background rate of about 0.5 , canceling the assumed latitude correction. In practice, the invisible muon decay rate will be measured and the assumed finalstate probabilities checked. Our estimated residual invisible muon decay background rate in SNO of 0.5/kton/yr in the range $20-30 \mathrm{MeV}$ is conservative, and will likely be reduced by several corrections we have neglected, each at the $\sim 10-20 \%$ level and favorable. These include: a smaller latitude correction would be more accurate, the number of oxygen nuclei per kton of heavy water is less than in light water, $\mu^{-}$capture on oxygen will reduce the number of background invisible muon decays, and the neutrino-deuteron cross sections are less than for free nucleons. Also, the observing period in SNO is mostly after that used in the SK analysis [4, and closer to solar maximum, when atmospheric neutrino fluxes are smaller, particularly at high latitudes and low energies [41].

\section{SNO Sensitivity to the Electron Neutrino Flux}

To estimate the SNO sensitivity to DSNB $\nu_{e}$ in the energy interval $20-30 \mathrm{MeV}$, we consider two primary requirements. First, that in the assumed 5 kton-yr exposure, there should be at least one signal event. Second, that the number of signal events $N_{S}$ should be larger than the square root of the number of background events $N_{B}$ for the same exposure (after our assumed cuts). Since we estimate $N_{B}=2.5$, the second requirement $N_{S}>\sqrt{N_{B}}=1.6$ is hardly different from the first that $N_{S}>1$. Our treatment is crude, but it is not yet possible to be more precise. The SNO backgrounds rates, which we have tried to estimate conservatively (see above), have not yet been published. Further, since both $N_{S}, N_{B} \sim 1$, the statistical fluctuations in each will also be $\sim 1$, and what numbers of events happen to occur will affect the final conclusions. In addition, the energies at which they occur will matter - a single event at $21 \mathrm{MeV}$ would be interpreted differently than a single event at $29 \mathrm{MeV}$. Since the statistical errors are so large, we can neglect all other uncertainties. In trying to motivate a complete and sophisticated analysis of the SNO data, we are simply trying to establish the likely scale of the sensitivity and show why this would be an interesting and important result. The full analysis will have to be done by the SNO Collaboration.

Since the sensitivity is above the level of standard predictions, we consider how much larger the flux would have to be to yield a detection. This factor must be at least

$$
\frac{\phi_{\text {sens. }}}{\phi_{\text {pred. }}} \simeq \frac{\max \left(1, \sqrt{N_{B}}\right)}{N_{S}}
$$

where inside this equation, $N_{S}$ is the number of signal events predicted in the standard case. Since $N_{S}, N_{B}$ are so small, we neglect the possible spectral differences between signal and background in the range $20-30 \mathrm{MeV}$, and only consider the counts, so that the required $N_{S}$ does not depend on the assumed supernova neutrino temperature. Since the predicted flux in this range does depend on temperature, so does the required renormalization in order to have a detection. These results are given in Table $\llbracket$ as a function of the assumed temperature. For low temperatures, large fluxes would be required. On the other hand, for larger temperatures, the SNO sensitivity is reasonably close to standard predictions. We do not consider neutrino mixing for three reasons. First, the low statistics. Second, the fact that over this narrow energy interval, a composite spectrum could be considered to be dominated by a largest temperature contributing to the spectrum. Third, since the estimated sensitivity is larger than standard predictions, any discovery would mean that our understanding of supernovae was missing an effect more significant than neutrino mixing.

The estimated sensitivity can also be approximately characterized by the required flux in this interval, instead of the number of events. Multiplying the flux, the number of deuterons, the cross section at $25 \mathrm{MeV}$, and the assumed 5-year exposure, we find $\phi\left(6 \times 10^{31}\right)(27 \times$ $\left.10^{-42} \mathrm{~cm}^{2}\right)(5$ yrs $) \simeq 1.6$ events, so that the required flux is $6 \mathrm{~cm}^{-2} \mathrm{~s}^{-1}$. Ignoring the slight temperature dependence, we show this as a constant in Fig. 3.

\section{ELECTRON NEUTRINOS FROM SN 1987A}

The neutrino flux from SN 1987A was observed with two water Čerenkov detectors, Kamiokande-II (Kam-II) and IMB 42, 43, 44, 45]. (And likely also with the much smaller Baksan scintillator detector, which had no directional sensitivity [46].) The most detectable component is the $\bar{\nu}_{e}$ flux, due to the largest cross section being the inverse beta reaction $\bar{\nu}_{e}+p \rightarrow e^{+}+n$, in which the outgoing positron angular distribution is nearly isotropic [25]. 
Two features of the measured angular data remain mysterious. One is that the first event in Kam-II was directed forward. The other is that the majority of the 12 Kam-II events, and all but one of the 8 IMB events, were in the forward hemisphere. Assuming that the yield is dominated by inverse beta events, both features, and especially the latter, are unlikely. These features suggest a larger than expected contribution from $\nu+e^{-} \rightarrow \nu+e^{-}$ scattering, in which the electron closely follows the neutrino direction. If so, then the most likely possibility is that this was caused by an enhanced flux of $\nu_{e}$; the $\bar{\nu}_{e}$ flux is constrained by the inverse beta yield, and the other flavors have $\sim 6$ times smaller cross sections on electrons.

Taking the measured data at face value, a few interpretations of the noted features are possible. One, that the $\nu_{e}$ flux in the early neutronization burst phase was much larger than expected. This could explain the first forward event, but not the time-averaged angular distribution. Two, that the $\nu_{e}$ flux in the longer thermal phase was much larger than expected. This could help explain the time-averaged angular distribution, and if the flux were large enough, also make it likely that one of the $\nu_{e}+e^{-} \rightarrow \nu_{e}+e^{-}$events would happen to be the first event detected. Third, that there were statistical fluctuations, either upward fluctuations of the small expected yields of $\nu_{e}+e^{-} \rightarrow \nu_{e}+e^{-}$scattering, or forward fluctuations of the near-isotropic angular distribution of $\bar{\nu}_{e}+p \rightarrow e^{+}+n$. With the SN 1987A data alone, it not possible to decide among these interpretations. In the context of standard supernova models, the first two interpretations are disfavored. What we consider here is that the required enhancements to the $\nu_{e}$ fluxes in the first two interpretations would similarly increase the expectations for the DSNB $\nu_{e}$ flux, and that this should be testable with SNO.

At Kam-II the first event was perfectly forward, with an angle $18 \pm 18$ degrees relative to the neutrino direction. During the neutronization phase, a prompt $\nu_{e}$ burst is created from electron captures on newly-liberated protons, $e^{-}+p \rightarrow \nu_{e}+n$ [47, 48, 49, 50, 51]. The fact that the first event was both forward and first is suggestive of its being caused by neutrino-electron scattering [52], whatever the difficulties of explaining this with standard supernova models [14, 47, 53]. (The relative timing of the Kam-II and IMB detectors is unknown, and we are making the common assumption that the first event in Kam-II was the first event overall.) The total energy released during this burst is expected to be about an order of magnitude less than the $\nu_{e}$ energy released during the thermal phase. For a total $\nu_{e}$ burst energy of $4.5 \times 10^{51}$ erg [4], the expected number of $\nu_{e}$ scatterings at KamII is $\sim 0.01$ events, compared to $\sim 0.1$ for the thermal phase. These estimates neglect neutrino mixing, which can be especially relevant for the burst phase, since the transformation of $\nu_{e}$ into other flavors occurs without the reverse process [50, 54, 55].

An enhanced rate of $\nu_{e}+e^{-} \rightarrow \nu_{e}+e^{-}$during the thermal phase could help explain why the time-averaged an-

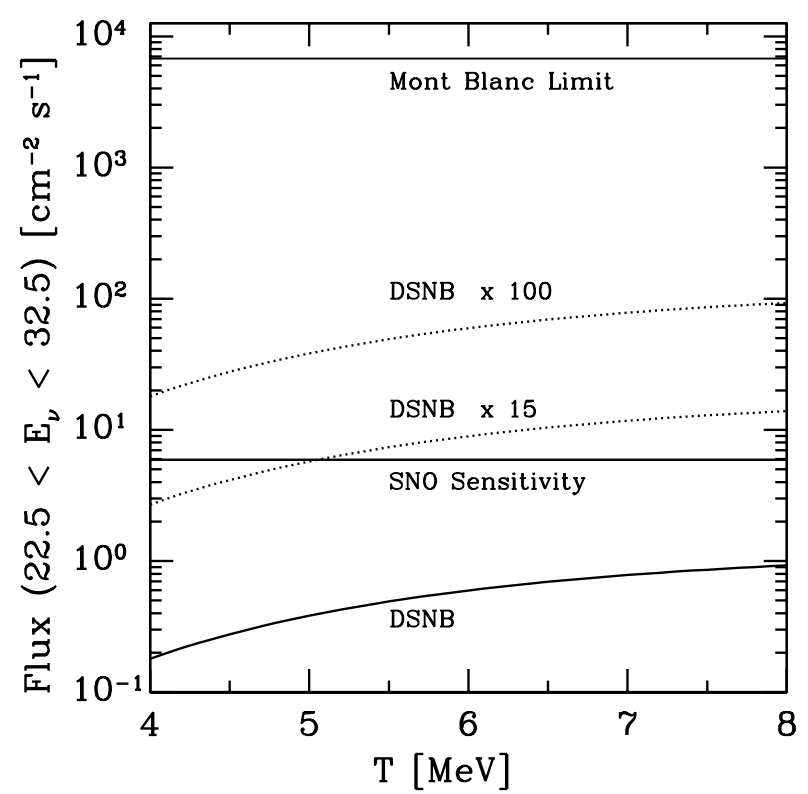

FIG. 3: The predicted DSNB flux as a function of the $\nu_{e}$ temperature. The approximate Mont Blanc limit and projected SNO sensitivity are shown with solid lines. The dotted lines approximately indicate the enhanced DSNB $\nu_{e}$ fluxes expected if the apparent indications of $\nu_{e}+e^{-} \rightarrow \nu_{e}+e^{-}$events in the SN 1987A data are interpreted as a probable outcome. The upper dotted line corresponds to assuming that the first Kam-II event was due to $\nu_{e}$ from the neutronization burst phase. The lower dotted line corresponds to assuming instead that it arose from $\nu_{e}$ from the thermal phase (and that in total $\sim 3$ of the events in Kam-II were due to $\nu_{e}$ ).

gular distributions were so forward [56, 57]. A recent reanalysis determines that a few of the Kam-II events, and at most one of the IMB events, are consistent with elastic scattering events [58]. With or without these events, the angular distributions of Kam-II and IMB are not consistent with each other (or with expectations), when one considers both the mean and the variance of $\cos \theta[25,58]$. However, as a caution about the small-number statistics, note that had even a single additional event been detected in a backward direction in IMB, then the disagreements would have been less severe 25].

Some potential scenarios for increased $\nu_{e}$ emission have been considered in Refs. [58, 59, 60, 61]. A boost in the $\nu_{e}$ yield relative to $\bar{\nu}_{e}$ can be characterized by the factor

$$
f_{\nu_{e}}=\frac{n}{N-n} \frac{\left\langle\sigma_{\bar{\nu}_{e}}\right\rangle / T_{\bar{\nu}_{e}}}{\left\langle\sigma_{\nu_{e}}\right\rangle / T_{\nu_{e}}} .
$$

Here $N$ is the total number of events observed at Kam-II or IMB (12 or 8), $n$ is the number of $\nu_{e}+e^{-} \rightarrow \nu_{e}+e^{-}$ scatterings, and $\langle\sigma\rangle$ is the thermally averaged crosssection, taking into account the thermal distribution and the efficiency of the detector [62]. In deriving Eq. (6), we have assumed that there is equal total energy emitted in each neutrino flavor. Since during the burst phase the total energy emitted in $\nu_{e}$ is approximately an order of 
magnitude less than by $\nu_{e}$ in the thermal phase, $f_{\nu_{e}}$ increases by $E_{\bar{\nu}_{e}}^{\text {thermal }} / E_{\nu_{e}}^{\text {burst }}$ when considering a probable scenario of one event during the burst phase.

For an illustration for a typical value of $f_{\nu_{e}}$ for the thermal phase we consider $n=3$ and $T_{\bar{\nu}_{e}}=3 \mathrm{MeV}$ for Kam-II. We choose $n=3$ since this is the smallest $n$ such that there is a reasonable chance of having an electron scattering event be first, and also so that the timeintegrated angular distribution is affected at a significant level. A typical value is $f_{\nu_{e}} \sim 15$ over the range of $T_{\nu_{e}}$. In Fig. [3 we show what this increase corresponds to in the DSNB flux, and how it compares to the SNO sensitivity. (If the $\nu_{e}$ is assumed to come from the neutronization burst phase, the required boost is even larger, at least $\sim 100$.) At high assumed temperatures, the suggested $\nu_{e}$ flux enhancements in the thermal phase would lead to too many charged-current events on ${ }^{16} \mathrm{O}$ in the SN 1987A data, which would be an independent way to constrain these scenarios. For example, for standard fluxes, this yield would become a few events when $T=8 \mathrm{MeV}$; in addition, the angular distribution favors backward angles 62]. Also, for large temperatures and fluxes, the number of events expected in the Homestake detector might become too large [63].

There also remains the possibility that the first Kam-II event resulted from $\bar{\nu}_{e}+p \rightarrow e^{+}+n$, and just happened to be emitted in the forward direction. For example, there is a $\sim 5 \%$ chance that any $\bar{\nu}_{e}$ event was emitted within a given cone of half-angle 25 degrees, comparable to the uncertainty in the Kam-II angular resolution. To have the first event be forward thus has a $\sim 5 \%$ probability, and to explain the time-averaged angular distribution would require additional statistical fluctuations. By searching for the DSNB $\nu_{e}$ flux, SNO will be the first experiment able to revisit the electron-neutrino component of the SN 1987A data and determine if the observed angular distribution was indeed a probable outcome.

\section{CONCLUSIONS}

We have shown that SNO should have sensitivity to a Diffuse Supernova Neutrino Background (DSNB) $\nu_{e}$ flux as low as $\simeq 6 \mathrm{~cm}^{-2} \mathrm{~s}^{-1}$, which is about three orders of magnitude smaller than the current limit from the Mont Blanc experiment [12]. In order to best avoid detector backgrounds, this is the flux just in the energy range 22.5-32.5 MeV; our corresponding theoretical predictions, given in Table【 are $\lesssim 1 \mathrm{~cm}^{-2} \mathrm{~s}^{-1}$. Our results depend on our estimates of the relevant solar and atmospheric neutrino backgrounds, and how the latter might be reduced. To the extent possible, we based our estimates on measured rates, especially the sub-Čerenkov muon decay rate from SK. Given the very small projected statistics, a more precise estimate of the sensitivity is not yet possible. A full analysis by the SNO Collaboration, using the measured data, is strongly encouraged.

Our estimate for the SNO sensitivity to DSNB $\nu_{e}$ may seem surprisingly good, given that SNO is much smaller than SK. However, it can be confirmed by adjusting the existing SK DSNB $\bar{\nu}_{e}$ limit of $1.2 \mathrm{~cm}^{-2} \mathrm{~s}^{-1}$ for the difference in exposure, ignoring the small differences in the considered energy ranges. The SK exposure was 22.5 kton for 4.1 years at about $50 \%$ efficiency in the relevant energy range, so about 45 kton-yr at full efficiency, or about 9 times more than the assumed SNO exposure. Since the SK limit arises from a background-limited search, the scaling to 5 kton-yr in a light water detector would worsen the limit by about $\sqrt{9}=3$. Taking into account the smaller signal cross section in a heavy water detector (and assuming the same background rate) gives an additional penalty of a factor $\simeq 2$, so that in the end the SNO sensitivity should be about a factor of 6 worse than in SK, close to what we deduced. (Had SK been rate-limited, the estimated SNO sensitivity would have instead been about 18 times worse.) Note that if the true SNO exposure is less than the 5 years of full efficiency that we assumed, the flux sensitivity only changes by the square root of the ratio of exposures.

An interesting related point is that once the KamLAND and SNO detectors report on multiple kton-yr exposures, they should be able to set DSNB $\bar{\nu}_{e}$ limits only a factor of a few to several weaker than what SK has published so far. This would be significant principally because KamLAND and SNO should have lower backgrounds and thresholds; the combination of these limits with the stronger limits at higher energies in SK might lead to improved limits on the supernova rates at higher redshift 7, 30]. The KamLAND and SNO limits so far [31, 64] are significantly worse than we are estimating here because they were based on the small exposures of early data and considered narrower energy ranges. In addition, the SNO analysis was based on the pure $\mathrm{D}_{2} \mathrm{O}$ phase, during which the neutron detection efficiency was much lower than in the next two phases. The sensitivity improves linearly with the exposure before the background-dominated regime is reached, after which it improves only with the square root. In terms of absolute sensitivity, the signal requirements demand an exposure of at least several kton-yr, beyond which we estimate that SNO will be background-dominated. (On a related issue, note that while the present SK DSNB $\bar{\nu}_{e}$ sensitivity is background-limited, if SK is enhanced by the addition of gadolinium, then the absolute sensitivity will be greatly improved, and is projected to become rate-limited [9].)

Studying the DSNB with any $\nu_{e}$ channel other than $\nu_{e}+d$ requires a large cross section, large detector mass, and favorable kinematics. In the future, this may be possible with large liquid argon detectors, as long emphasized by Cline [65]. For example, the estimated DSNB $\nu_{e}$ sensitivity after a 15 kton-yr exposure of the ICARUS detector is $\simeq 1.6 \mathrm{~cm}^{-2} \mathrm{~s}^{-1}[\underline{66}$, which would be very interesting as a complement to the SK DSNB $\bar{\nu}_{e}$ sensitivity. This estimate assumes that the only backgrounds in ICARUS are those due to solar and atmospheric $\nu_{e}$. Very 
likely, other backgrounds will have to be considered as well. For example, it is assumed that the low-momentum (sub-Čerenkov) muons, which are a serious background in water-based detectors, could be completely rejected in argon-based detectors [65, 66]. In water-based detectors, the muons with momenta below $\simeq 100 \mathrm{MeV}$ are invisible. In a liquid argon detector with an assumed kinetic energy threshold of $5 \mathrm{MeV}$, muons with momenta less than $\simeq 30$ $\mathrm{MeV}$ would also be invisible, and their decays would produce a relevant background. Also needed are studies of backgrounds induced by fast neutrons entering the detector, as well as those arising from the quenched light output of energetic charged particles. Nevertheless, this technique appears promising, and may have the potential to go significantly beyond the sensitivity of SNO.

Besides waiting for another Milky Way supernova, in principle there is another method to probe the $\nu_{e}$ emission. The $\nu_{e}$ flux from past Milky Way supernovae would have transformed terrestrial molybdenum isotopes into unstable technetium isotopes (half-lives 2.6 and 4.2 million years), and the elemental ratios in deep molybdenum deposits should reveal the received $\nu_{e}$ fluence over a comparable time period [67]. To the best of our knowledge, the realistic sensitivity of this technique is unknown, and no experiment is planned.

In addition to improving the present DSNB $\nu_{e}$ sensitivity by about three orders of magnitude, a full analysis by SNO can test whether the large $\nu_{e}$ flux suggested by the SN 1987A data was probable, or was simply a statistical fluctuation. If the first event in Kamiokande-II was really $\nu_{e}+e^{-} \rightarrow \nu_{e}+e^{-}$scattering, and was a probable outcome, then the DSNB $\nu_{e}$ flux should be greatly enhanced. If this first event is assumed to be from the neutronization phase, then the required enhancement is a factor at least $\simeq 100$. If this first event is assumed to be from the thermal phase, the required enhancement is a factor at least $\simeq 15$; this would also mean that there should be at least a few other $\nu_{e}$ events in the data, which would make the angular distributions more forward, also as suggested by the data. Both scenarios are probably unrealistic, and our estimates are crude. However, our point is that at present, only SNO has the sensitivity to directly constrain them, and for the first time be able to look back and shed light on the mysteries still lingering from SN 1987A.

\section{Acknowledgments}

We thank David Cline, Raph Hix, Josh Klein, Terry Walker, and especially Shin'ichiro Ando, Mark Vagins and Hasan Yüksel for helpful discussions. J. F. B. is supported by The Ohio State University, and L. E. S. by Department of Energy grant DE-FG02-91ER40690.
[1] A. Burrows, D. Klein and R. Gandhi, Phys. Rev. D 45, 3361 (1992); A. B. Balantekin and F. Loreti, Phys. Rev. D 45, 1059 (1992); J. F. Beacom and P. Vogel, Phys. Rev. D 58, 053010 (1998); J. F. Beacom and P. Vogel, Phys. Rev. D 58, 093012 (1998); L. Cadonati, F. P. Calaprice and M. C. Chen, Astropart. Phys. 16, 361 (2002); C. J. Virtue [SNO Collaboration], Nucl. Phys. Proc. Suppl. 100, 326 (2001); J. Ahrens et al. [AMANDA Collaboration], Astropart. Phys. 16, 345 (2002); M. K. Sharp, J. F. Beacom and J. A. Formaggio, Phys. Rev. D 66, 013012 (2002); J. F. Beacom, W. M. Farr and P. Vogel, Phys. Rev. D 66, 033001 (2002); F. Cei, Int. J. Mod. Phys. A 17, 1765 (2002); L. Miramonti, arXiv:hep-ex/0307029 A. Bandyopadhyay, S. Choubey, S. Goswami and K. Kar, arXiv:hep-ph/0312315 P. Antonioli et al., New J. Phys. 6, 114 (2004); M. R. Vagins, Nucl. Phys. Proc. Suppl. 143, 457 (2005); G. L. Fogli, E. Lisi, A. Mirizzi and D. Montanino, JCAP 0504, 002 (2005).

[2] S. van den Bergh, Phys. Rept. 204, 385 (1991); S. van den Bergh, Comments Astrophys. 17, 125 (1993); G. A. Tammann, W. Loeffler and A. Schroder, Astrophys. J. Suppl. 92, 487 (1994); R. G. Strom, Astron. Astrophys. 288, L1 (1994); P. M. Dragicevich, D. G. Blair, and R. R. Burman, Mon. Not. Roy. Astron. Soc. 302, 693 (1999); E. N. Alekseev and L. N. Alekseeva, J. Exp. Theor. Phys. 95, 5 (2002) [Zh. Eksp. Teor. Fiz. 95, 10 (2002)].

[3] S. Ando, J. F. Beacom and H. Yuksel,
arXiv:astro-ph/0503321

[4] M. Malek et al. [Super-Kamiokande Collaboration], Phys. Rev. Lett. 90, 061101 (2003).

[5] M. Fukugita and M. Kawasaki, Mon. Not. Roy. Astron. Soc. 340, L7 (2003).

[6] S. Ando and K. Sato, Phys. Lett. B 559, 113 (2003).

[7] L. E. Strigari, M. Kaplinghat, G. Steigman and T. P. Walker, JCAP 0403, 007 (2004).

[8] L. E. Strigari, J. F. Beacom, T. P. Walker and P. Zhang, JCAP 0504, 017 (2005).

[9] J. F. Beacom and M. R. Vagins, Phys. Rev. Lett. 93, 171101 (2004).

[10] M. R. Vagins (Principal Investigator), Department of Energy 2003 and 2005 Advanced Detector Research program grants; M. R. Vagins, talk at "NNN05: Next Generation of Nucleon Decay and Neutrino Detectors," 7-9 April 2005, Aussois, France, http://nnn05.in2p3.fr/

[11] S. N. Ahmed et al. [SNO Collaboration], Phys. Rev. Lett. 92, 181301 (2004); Q. R. Ahmad et al. [SNO Collaboration], Phys. Rev. Lett. 89, 011301 (2002); Q. R. Ahmad et al. [SNO Collaboration], Phys. Rev. Lett. 87, 071301 (2001).

[12] M. Aglietta et al., Astropart. Phys. 1, 1 (1992).

[13] C. Lunardini and A. Y. Smirnov, Nucl. Phys. B 616, 307 (2001); C. Lunardini and A. Y. Smirnov, JCAP 0306, 009 (2003).

[14] G. Raffelt, Stars as Laboratories of Fundamental Physics, The University of Chicago Press, 1996.

[15] A. Heger, C. L. Fryer, S. E. Woosley, N. Langer and 
D. H. Hartmann, Astrophys. J. 591, 288 (2003).

[16] M. T. Keil, G. G. Raffelt and H. T. Janka, Astrophys. J. 590, 971 (2003); T. A. Thompson, A. Burrows and P. A. Pinto, Astrophys. J. 592, 434 (2003); M. Liebendoerfer, M. Rampp, H. T. Janka and A. Mezzacappa, Astrophys. J. 620, 840 (2005); F. Cavanna, M. L. Costantini, O. Palamara and F. Vissani, Surveys High Energ. Phys. 19, 35 (2004); K. Sumiyoshi, S. Yamada, H. Suzuki, H. Shen, S. Chiba and H. Toki, arXiv:astro-ph/0506620

[17] B. Jegerlehner, F. Neubig and G. Raffelt, Phys. Rev. D 54, 1194 (1996); G. M. Fuller, W. C. Haxton and G. C. McLaughlin, Phys. Rev. D 59, 085005 (1999); A. S. Dighe and A. Y. Smirnov, Phys. Rev. D 62, 033007 (2000); H. Minakata, H. Nunokawa, R. Tomas and J. W. F. Valle, Phys. Lett. B 542, 239 (2002); A. S. Dighe, M. Kachelriess, G. G. Raffelt and R. Tomas, JCAP 0401, 004 (2004); G. L. Fogli, E. Lisi, A. Mirizzi and D. Montanino, Phys. Rev. D 70, 013001 (2004); A. B. Balantekin and H. Yuksel, New J. Phys. 7, 51 (2005); V. Barger, P. Huber and D. Marfatia, Phys. Lett. B 617, 167 (2005).

[18] A. M. Hopkins, Astrophys. J. 615, 209 (2004).

[19] D. Schiminovich et al, Astrophys. J. 619 L47 (2005).

[20] I. K. Baldry et al., Mon. Not. Roy. Astron. Soc. 358, 441 (2005).

[21] P. G. Perez-Gonzalez et al., arXiv:astro-ph/0505101

[22] E. F. Bell et al., Astrophys. J. 625, 23 (2005).

[23] J. F. Beacom, R. N. Boyd and A. Mezzacappa, Phys. Rev. D 63, 073011 (2001).

[24] S. Ando and K. Sato, New J. Phys. 6, 170 (2004).

[25] P. Vogel and J. F. Beacom, Phys. Rev. D 60, 053003 (1999).

[26] S. Nakayama et al. [K2K Collaboration], Phys. Lett. B 619, 255 (2005); E. Aliu et al. [K2K Collaboration], Phys. Rev. Lett. 94, 081802 (2005).

[27] J. Boger et al. [SNO Collaboration], Nucl. Instrum. Meth. A 449, 172 (2000).

[28] B. Aharmim et al. [SNO Collaboration], nucl-ex/0502021

[29] M. Kaplinghat, G. Steigman and T. P. Walker, Phys. Rev. D 62, 043001 (2000).

[30] S. Ando, K. Sato and T. Totani, Astropart. Phys. 18, 307 (2003).

[31] B. Aharmim et al. [SNO Collaboration], Phys. Rev. D 70, 093014 (2004).

[32] M. Butler, J. W. Chen and X. Kong, Phys. Rev. C 63, 035501 (2001).

[33] S. Nakamura, T. Sato, S. Ando, T. S. Park, F. Myhrer, V. Gudkov and K. Kubodera, Nucl. Phys. A 707, 561 (2002).

[34] J. N. Bahcall and M. H. Pinsonneault, Phys. Rev. Lett. 92, 121301 (2004).

[35] L. E. Marcucci, R. Schiavilla, M. Viviani, A. Kievsky and S. Rosati, Phys. Rev. Lett. 84, 5959 (2000); L. E. Marcucci, R. Schiavilla, M. Viviani, A. Kievsky, S. Rosati and J. F. Beacom, Phys. Rev. C 63, 015801 (2001); T. S. Park et al., Phys. Rev. C 67, 055206 (2003); K. Kubodera and T. S. Park, Ann. Rev. Nucl. Part. Sci. 54, 19 (2004).

[36] W. T. Winter et al., Phys. Rev. Lett. 91, 252501 (2003); W. T. Winter, S. J. Freedman, K. E. Rehm and J. P. Schiffer, arXiv:nucl-ex/0406019

[37] A. Odrzywolek, M. Misiaszek and M. Kutschera, Astropart. Phys. 21, 303 (2004); C. Porciani, S. Petroni and G. Fiorentini, Astropart. Phys. 20, 683 (2004); F. Iocco,
G. Mangano, G. Miele, G. G. Raffelt and P. D. Serpico, Astropart. Phys. 23, 303 (2005).

[38] V. A. Naumov, arXiv:hep-ph/0201310

[39] G. Barr, T. K. Gaisser and T. Stanev, Phys. Rev. D 39, 3532 (1989).

[40] E. Kolbe, K. Langanke and P. Vogel, Phys. Rev. D 66, 013007 (2002).

[41] T. K. Gaisser and M. Honda, Ann. Rev. Nucl. Part. Sci. 52, 153 (2002).

[42] K. Hirata et al. [KAMIOKANDE-II Collaboration], Phys. Rev. Lett. 58, 1490 (1987).

[43] K. S. Hirata et al., Phys. Rev. D 38, 448 (1988).

[44] R. M. Bionta et al., Phys. Rev. Lett. 58, 1494 (1987).

[45] C. B. Bratton et al. [IMB Collaboration], Phys. Rev. D 37, 3361 (1988).

[46] E. N. Alekseev, L. N. Alekseeva, V. I. Volchenko and I. V. Krivosheina, JETP Lett. 45, 589 (1987) [Pisma Zh. Eksp. Teor. Fiz. 45, 461 (1987)]; E. N. Alekseev, L. N. Alekseeva, I. V. Krivosheina and V. I. Volchenko, Phys. Lett. B 205, 209 (1988).

[47] S. W. Bruenn, Phys. Rev. Lett. 59, 938 (1987).

[48] M. Rampp and H. T. Janka, Astrophys. J. 539, L33 (2000).

[49] A. Mezzacappa, M. Liebendoerfer, O. E. B. Messer, W. R. Hix, F. K. Thielemann and S. W. Bruenn, Phys. Rev. Lett. 86, 1935 (2001).

[50] M. Kachelriess, R. Tomas, R. Buras, H. T. Janka, A. Marek and M. Rampp, astro-ph/0412082

[51] F. K. Sutaria and A. Ray, Phys. Rev. Lett. 79, 1599 (1997).

[52] J. N. Bahcall, T. Piran, W. H. Press and D. N. Spergel, Nature 327, 682 (1987); J. Arafune and M. Fukugita, Phys. Rev. Lett. 59, 367 (1987); D. Notzold, Phys. Lett. B 196, 315 (1987); A. V. Barnes, S. Pakvasa and T. J. Weiler, Astrophys. J. 323, L31 (1987); S. P. Rosen, Phys. Rev. D 37, 1682 (1988); H. Murayama and T. Yanagida, Phys. Lett. B 520, 263 (2001).

[53] K. Sato and H. Suzuki, Phys. Rev. Lett. 58, 2722 (1987).

[54] T. P. Walker and D. N. Schramm, Phys. Lett. B 195, 331 (1987).

[55] S. Ando, Phys. Rev. D 70, 033004 (2004).

[56] J. M. LoSecco, Phys. Rev. D 39, 1013 (1989).

[57] D. Kielczewska, Phys. Rev. D 41, 2967 (1990).

[58] M. L. Costantini, A. Ianni and F. Vissani, Phys. Rev. D 70, 043006 (2004).

[59] D. W. Arnett, Astrophys. J. 319, 136 (1987).

[60] A. Burrows and J. M. Lattimer, Astrophys. J. 318, L63 (1987).

[61] V. S. Imshennik and O. G. Ryazhskaya, Astron. Lett. 30, 14 (2004).

[62] W. C. Haxton, Phys. Rev. D 36, 2283 (1987).

[63] H. Minakata, H. Nunokawa and K. Shiraishi, Phys. Rev. D 38, 694 (1988).

[64] K. Eguchi et al. [KamLAND Collaboration], Phys. Rev. Lett. 92, 071301 (2004).

[65] D. B. Cline, arXiv:astro-ph/0001237

[66] A. G. Cocco, A. Ereditato, G. Fiorillo, G. Mangano and V. Pettorino, JCAP 0412, 002 (2004).

[67] G. A. Cowan and W. C. Haxton, Science 216, 51 (1982); W. C. Haxton and C. W. Johnson, Nature 333, 325 (1988); V. T. Nguyen and C. W. Johnson, arXiv:astro-ph/0508267 